\title{
Correction to: Gaussian mixture model-based cluster analysis of apparent diffusion coefficient values: a novel approach to evaluate uterine endometrioid carcinoma grade
}

\author{
Sakiko Kageyama ${ }^{1} \cdot$ Naoko Mori $^{1}$ (D) $\cdot$ Shunji Mugikura ${ }^{1,2} \cdot$ Hideki Tokunaga $^{3} \cdot$ Kei Takase $^{1}$ \\ Published online: 9 September 2020 \\ (C) European Society of Radiology 2020
}

\section{Correction to European Radiology}

https://doi.org/10.1007/s00330-020-07047-6

The original version of this article, published on 28 July 2020 , unfortunately contained a mistake. The presentation of Figure 2 was incorrect. The corrected Figure 2 is given below.

Publisher's note Springer Nature remains neutral with regard to jurisdictional claims in published maps and institutional affiliations.

The online version of the original article can be found at https://doi.org/ 10.1007/s00330-020-07047-6

Naoko Mori

naokomori7127@gmail.com

1 Department of Diagnostic Radiology, Tohoku University Graduate School of Medicine, Seiryo 1-1, Sendai 980-8574, Japan

2 Department of Image Statistics, Tohoku Medical Megabank Organization, Tohoku University, Seiryo 2-1, Sendai 980-8574, Japan

3 Department of Obstetrics and Gynecology, Tohoku University Graduate School of Medicine, Seiryo 1-1, Sendai 980-8574, Japan 


\section{(a) Training cohort of observer 1}

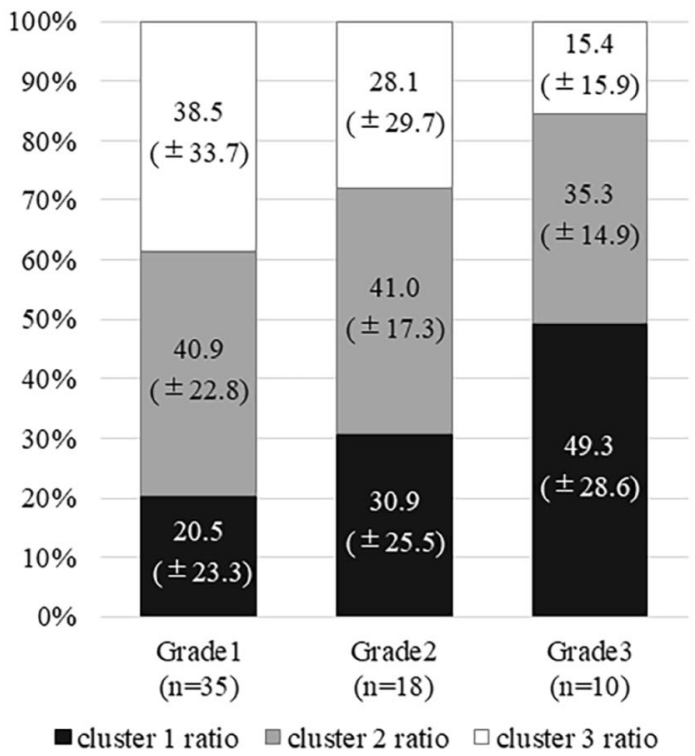

Fig. 2 Graph of the cluster ratios calculated within each grade in the training cohort (a) and validation cohort (b) by observer 1 . The cluster ratios show a significant association with histological grade. There was a significant positive correlation between the cluster 1 ratio and histological

\section{(b) Validation cohort of observer 1}

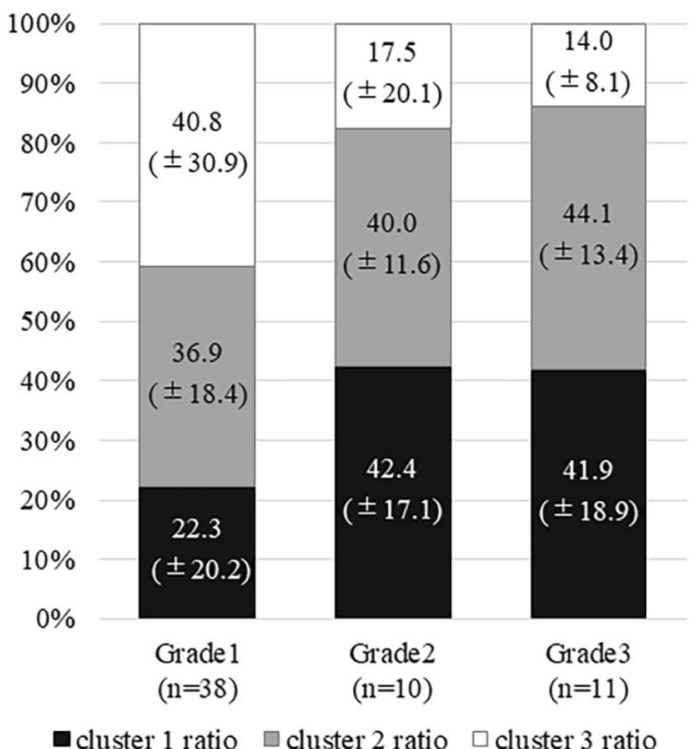

grade in both training and validation cohorts; the higher the grade, the higher the cluster 1 ratio was. There was a significant negative correlation between the cluster 3 ratio and histological grade in both training and validation cohorts; the higher the grade, the lower the cluster 3 ratio 